\title{
ORIGINAL
}

\section{PRESENTACIÓN DE LA COHORTE "CDC DE CANARIAS": OBJETIVOS, DISEÑO Y RESULTADOS PRELIMINARES (*)}

\author{
Antonio Cabrera de León (1,2,3), $\mathrm{M}^{\mathrm{a}}$ del Cristo Rodríguez Pérez (1,4), Delia Almeida González (1), \\ Ana González Hernández (1,5), Lina I. Pérez Méndez $(1,4)$ y el grupo CDC* \\ (1) Unidad de Investigación. Hospital Universitario Ntra. Sra. de La Candelaria. Tenerife. \\ (2) Hospital San Juan de Dios. Tenerife. \\ (3) Universidad de La Laguna. Tenerife. \\ (4) Gerencia de Atención Primaria. Tenerife. \\ (5) Instituto Canario de Investigación del Cáncer.
} Santiago Domínguez Coello (1,4), Armando Aguirre Jaime (1,4), Buenaventura Brito Díaz $(1,4)$,

\section{RESUMEN}

Fundamento: Canarias ocupa el primer lugar de España en mortalidad por cardiopatía isquémica y por diabetes. Sus mujeres son las primeras en mortalidad por cáncer de mama. El "CDC de Canarias" es el estudio de una cohorte de población general para analizar la prevalencia e incidencia de estas enfermedades y la exposición a sus factores de riesgo (FR) en la población adulta del archipiélago.

Métodos: Estudio prospectivo con muestreo aleatorio en población general, en el que participaron 6.729 personas entre los años 2000 y 2005 (edad 18-75 años). Se les realizó antropometría y se extrajo sangre para almacenamiento de muestras séricas y genéticas. Mediante cuestionario se recogió: hábitos dietéticos, actividad física, antecedentes personales y familiares de enfermedad, exposición a FR laborales o ambientales, tabaquismo, etc.

Resultados: La prevalencia de obesidad es casi del $30 \%$, sin diferencias entre sexos, pero el sobrepeso afecta más a los varones (45 vs. $33 \%$; p<0'001), los cuales presentan también mayor prevalencia de diabetes ( 12 vs. $10 \%$; $\mathrm{p}=0$ '005), hipertensión (43 vs. 33\%; p<0'001), ingesta excesiva de alcohol (13 vs. $2 \%$; $\mathrm{p}<0$ ’001) y falta de protección solar (46 vs. 18\%; p<0’001). En las mujeres es más frecuente la exposición a bajos niveles de colesterol HDL (37 vs. 30\%; p<0'001) y al sedentarismo (71 vs $55 \%$; $<<0$ '001). La exposición a los FR estudiados, entre ellos la pobreza, es mayor en edades avanzadas, salvo el tabaquismo (26\%) que es mayor en edades jóvenes. La estimación de riesgos relativos de exposición a los factores de riesgo cardiovascular y de cáncer es más alta en las clases sociales pobres.

Conclusiones: La actual población adulta de Canarias presenta una elevada prevalencia de exposición a factores de riesgo para las enfermedades cardiovasculares, la diabetes y el cáncer, destacando especialmente el sobrepeso, la obesidad y el sedentarismo.

Palabras claves: Estudio de cohorte. Cáncer. Diabetes mellitus. Enfermedades cardiovasculares. Factores de riesgo.

Correspondencia:

Antonio Cabrera de León

Hospital San Juan de Dios.

Carretera Santa Cruz - La Laguna, 53

38009. Santa Cruz de Tenerife

Correo electrónico: acabrera@sjd.es

\section{ABSTRACT}

\section{Presentation of the "CDC de Canarias" Cohort: Objectives, Design and Preliminary Results}

Background: The Canary Islands rank first in Spain with respect to the ischaemic heart disease and diabetes mortality rates. The Islands' female population leads the country in deaths from breast cancer. The "CDC de Canarias" is a general population cohort study in order to analyse the prevalence and incidence of these diseases and the exposure to their risk factors (RF) in the adult population of the archipelago.

Methods: Prospective study with a random sampling of the general population, in which 6,729 individuals participated between 2000 and 2005 (aged 18-75). Anthropometric measurements were taken, and blood was drawn for the storage of serum and genetic samples. The following information was gathered through a questionnaire: eating habits, physical activity, personal and family medical history, exposure to occupational or environmental risk factors, smoking, etc.

Results: The prevalence of obesity is close to $30 \%$, without differences between sexes, however, more male subjects were overweight than women ( $45 \mathrm{vs} .33 \% ; \mathrm{p}<0.001)$ and also presented a greater prevalence of diabetes ( 12 vs. $10 \%$; $=0.005$ ), high blood pressure ( 43 vs. $33 \%$; $<<0.001$ ), excessive intake of alcohol (13 vs. $2 \%$; $p<0.001)$ and lack of sun protection (46 vs. $18 \%$; $<0.001$ ). Exposure to low levels of HDL cholesterol is more frequent in women ( 37 vs. $30 \% ; \mathrm{p}<0.001)$ as is also the case with a sedentary life style ( 71 vs. $55 \%$; $p<0.001)$. The exposure to the risk factors studied, including poverty, is greater in advanced age groups, except for smoking $(26 \%)$ which is greater in the younger subjects. The estimate of relative risks of exposure to cardiovascular and cancer risk factors is higher in low-income social classes.

Conclusions: The current adult population of the Canaries presents a high prevalence of exposure to risk factors for cardiovascular disease, diabetes and cancer, among which overweightness, obesity and lack of exercise stand out particularly.

Keywords: Cohort study. Cáncer. Diabetes mellitus. Cardiovascular Diseases. Risk factors.

(*) Financiación: Fondo de Investigaciones Sanitarias (99/0361, 02/1158, 02/1189, 07/0934) y la Fundación Canaria para la Investigación y Salud (45/98).

Los restantes miembros del Grupo CDC son: Carlos Borges Álamo, Lourdes Carrillo Fernández, José Carlos del Castillo Rodríguez, Basilio Anía Lafuente, Hilaria González Camacho, Noelia Fernández Ramos, Francisco Hernández Díaz y José Juan Alemán Sánchez. 


\section{INTRODUCCIÓN}

El conocimiento actual de los factores de riesgo (FR) frente a la enfermedad cardiovascular, la diabetes mellitus y el cáncer se ha obtenido principalmente del seguimiento de cohortes en Norteamérica y Europa del Norte ${ }^{1-4}$. Las poblaciones de Europa Meridional quedaron durante mucho tiempo al margen de tales estudios, por lo cual factores presentes en ellas no fueron conocidos hasta 30 años más tarde ${ }^{5}$ y no se han asentado definitivamente hasta años recientes ${ }^{6}$. Hoy en Europa existen grandes estudios de cohortes, como el proyecto $\mathrm{EPIC}^{7-8}$, en el que participan poblaciones del Norte y Sur, pero sigue siendo necesario el seguimiento de otras poblaciones que no han sido nunca objeto de ello, particularmente al Sur y al Este del continente. En España, ha habido hasta la fecha pocos estudios de seguimiento de poblaciones; además de aquellas integradas en el proyecto EPIC para el estudio del cáncer ${ }^{9}$, cabe mencionar la cohorte REGICOR ${ }^{10}$ para el estudio de riesgo cardiovascular, en concreto, de cardiopatía isquémica o la pequeña cohorte del Estudio de Asturias sobre incidencia de diabetes ${ }^{11}$. Otras cohortes como la del estudio SUN $^{12}$ y la de MANRESA ${ }^{13}$ sobre enfermedad y mortalidad cardiovascular no fueron reclutadas de entre la población general, por lo que presentan alguna limitación para la generalización de sus resultados a la misma.

En Canarias, frontera suroeste de la Unión Europea, nunca se ha estudiado una cohorte representativa de su población, que en su gran mayoría es culturalmente europea y étnicamente caucasiana, con permanencia genética de la población aborigen ${ }^{14}$. Actualmente sus enfermedades más importantes son las cardiovasculares, el cáncer y la diabetes mellitus ${ }^{16}$. De hecho, estas islas encabezan la mortalidad nacional por cardiopatía isquémica ${ }^{17}$ y diabetes ${ }^{18}$ y están entre las primeras Comunidades en morta- lidad por cáncer ${ }^{18}$. Se ha descrito que estas tasas pueden estar sobrevaloradas al atribuir a Canarias la mortalidad del gran volumen de turistas que visitan el archipiélago $^{19}$. Pero este posible sesgo no explicaría las diferencias que se observan entre las dos provincias que lo componen, ni tampoco las encontradas entre ésta y otras Comunidades españolas de similar economía turística.

Las mujeres de Gran Canaria padecen la mayor incidencia $\left(65 / 10^{5}\right)$ y mortalidad $\left(25 / 10^{5}\right)$ por cáncer de mama en España ${ }^{20}$. El cáncer broncopulmonar crecerá rápidamente en las mujeres canarias porque en la actual generación de adolescentes la proporción de chicas fumadoras casi duplica a la de los $\operatorname{chicos}^{21}$.

En cuanto a la diabetes tipo 2, los pocos estudios que han estandarizado la estructura etaria, han comprobado que muestra en las islas una prevalencia muy elevada ${ }^{22}$ y que el síndrome metabólico, conocido precursor de la diabetes, presenta una prevalencia en torno al $25 \%{ }^{23,24}$.

El estudio "CDC de Canarias" efectúa el seguimiento de una cohorte con la finalidad principal de estimar la prevalencia e incidencia de cáncer, diabetes y enfermedades cardiovasculares en la población adulta de las Islas Canarias, e identificar la exposición a FR. Las iniciales de estas tres enfermedades forman el acrónimo "CDC". El presente artículo tiene como objetivo la descripción del diseño, procedimientos de reclutamiento y medición de la cohorte, así como la presentación de algunos resultados preliminares sobre prevalencia.

\section{SUJETOS Y MÉTODOS}

Selección de la muestra: La cohorte "CDC de Canarias" se recogió de forma aleatoria a partir del Censo de tarjetas 
sanitarias, que incluye a la casi totalidad de la población residente en el archipiélago. La estrategia de muestreo fue de estratificación polietápica, con la isla como primera etapa y la comarca (norte y sur en cada isla) como segunda, seleccionando aleatoriamente al menos un municipio por comarca y realizando finalmente un muestreo aleatorio simple en cada municipio. Un tamaño mínimo de 5.500 personas permitiría, a 20 años de seguimiento, detectar 120 casos nuevos casos de cáncer, a partir de una tasa de incidencia esperada de $11 \times 10^{4}$ año $^{-1}$, una de las tasas más bajas de los tres problemas de salud abordados. En este cálculo se consideró que la tasa de respuesta alcanzaría, al menos, el 65\%. La participación alcanzó finalmente el $70 \%$, calculado como el porcentaje de participantes que acudió a la cita respecto al total de aquellos a los que se envió la segunda carta, una vez descontadas las cartas que fueron devueltas.

Como pilotaje previo al reclutamiento de la cohorte, en el año 1993 se estudió un conjunto de 612 residentes en la isla de El Hierro, cuyos principales resultados han sido publicados ${ }^{25}$. Esto hace que la muestra de población enrolada en esta isla tenga mayor tiempo de seguimiento que el resto de la cohorte y sea algo más vieja en edad. Otra peculiaridad ocurre con la variable sexo en la isla de Gran Canaria, donde la estrategia de muestreo se diseñó para reclutar una mayor proporción de mujeres que de hombres para disponer de potencia suficiente en el estudio del cáncer de mama, que presenta la mayor mortalidad de España en esta isla.

Este estudio fue aprobado previamente por el Comité de Bioética del Hospital Universitario Nuestra Señora de La Candelaria (HUNSC) y tanto el reclutamiento inicial como el seguimiento y posteriores estudios anidados han sido financiados por el Fondo de Investigaciones Sanitarias
(99/0361, 02/1158, 02/1189, 07/0934) y la Fundación Canaria para la Investigación y Salud (45/98).

Criterios de inclusión y exclusión: Los criterios para que una persona pudiera ser incluida en la cohorte CDC fueron: 1) figurar en el censo de tarjetas sanitarias, 2) tener entre 18 y 75 años, y 3) dar su consentimiento informado. Este último documento informó al posible participante sobre los objetivos del estudio, las pruebas a las que se le iba a someter, los contactos periódicos en años posteriores, y recabó su autorización para que los investigadores pudiesen: a) consultar su historial clínico, b) almacenar sus muestras séricas, hemáticas y genéticas, c) emplear toda la información así obtenida en estudios posteriores que fueran autorizados por el citado Comité de Bioética del HUNSC. El único criterio de exclusión fue la incapacidad de responder por sí mismo al encuestador y no disponer de un tutor que lo hiciese en su lugar en la primera entrevista.

Reclutamiento: comenzó en enero de 2000 y finalizó en diciembre de 2005, obteniéndose un tamaño muestral final de 6.729 participantes. El trabajo de campo fue efectuado por personal contratado exclusivamente para el estudio. La estrategia de reclutamiento comenzó recabando el apoyo de las autoridades sanitarias de cada isla, de las autoridades políticas del municipio elegido y de los equipos de Atención Primaria que atendían a la población seleccionada, a los cuáles se les pidió que manifestaran una opinión positiva en el caso de que algún paciente resultase seleccionado y les pidiera información sobre el estudio. A continuación, se divulgó el estudio en los medios de comunicación locales y luego se realizó un primer envío postal a los sujetos seleccionados con información sobre los objetivos del proyecto y pidiéndoles su participación. En un segundo envío postal, efectua- 
do quince días más tarde, se citó a los seleccionados para que acudieran en ayunas al centro de salud más cercano a su domicilio.

En el primer contacto se tomaron los datos identificativos y, tras la firma del consentimiento informado, una enfermera les realizó una exploración física y extracción de sangre. Asimismo, se acordó una segunda cita para que respondieran al cuestionario CDC (disponible en www.icic.es/cuestionario-CDC/docs), en una entrevista de una hora aproximada de duración, ofreciéndoles la máxima accesibilidad al darles a elegir día, lugar y hora de la entrevista.

Mediciones: Los datos identificativos fueron: nombre, número de documento nacional de identidad y de la seguridad social, domicilio, teléfonos, fecha y lugar de nacimiento, tiempo de residencia en Canarias, centro de salud al que está adscrito y nombre de su médico de cabecera. En la exploración se tomaron medidas antropométricas siguiendo las normas publicadas por SEEDO ${ }^{26}$. El participante debía estar descalzo, en ropa ligera y en bipedestación. Se obtuvo así, el peso (Kg), la talla, el perímetro abdominal, pelviano y de la muñeca (todos ellos en $\mathrm{cm}$ ). Con estas medidas se calculó el índice de masa corporal (IMC) en $\mathrm{kg} / \mathrm{m}^{2}$, la ratio abdomen/pelvis (RAP) y el índice abdomen/estatura (IAE). También se midió la frecuencia cardiaca y la presión arterial $(\mathrm{mmHg})$ siguiendo las recomendaciones de semFYC ${ }^{27}$. Aleatoriamente, al $10 \%$ de los participantes se les hizo un electrocardiograma basal.

Por último, se procedió a la extracción de $10 \mathrm{ml}$ de sangre venosa que se repartió en dos tubos: un tubo de bioquímica de 6 $\mathrm{ml}$ que fue centrifugado in situ a $2000 \mathrm{rpm}$ durante 10 minutos, a temperatura ambiente, tras no más de 1 hora de su extracción, para separar el suero del resto del material hemático; el otro contenía
EDTA para almacenar sangre total con una capacidad de $4 \mathrm{ml}$. Ambos tubos fueron conservados a $4^{\circ} \mathrm{C}$ en neveras portátiles que se trasladaron diariamente desde cada isla a Tenerife. Una vez en el HUNSC, se procesó el suero para la determinación de glucemia basal y fracciones lipídicas. El sobrante fue repartido en 4 alícuotas que se almacenaron a $-80^{\circ} \mathrm{C}$. $\mathrm{El}$ tubo que contenía sangre total se almacenó a $-20^{\circ} \mathrm{C}$, tras extracción de $\mathrm{ADN}$.

En la segunda cita a los participantes se les administró el cuestionario $\mathrm{CDC}$, que consta de 10 bloques de preguntas: A) Color de la piel, ojos y pelo y la presencia o no de manchas solares, nevus o pecas en zonas de exposición solar. B) Nivel educativo, actividad laboral, situación laboral, ingresos económicos. C) Antecedentes familiares de enfermedad y de consanguini$\mathrm{dad}^{28}$. D) Antecedentes personales y familiares de enfermedad siguiendo la codificación del CIE-9 MC y del CIE-10 29,30 y el consumo de medicamentos según la clasificación por principios terapéuticos ATC ${ }^{31}$. E) Exposición, tanto activa como pasiva, al tabaco. F) Historia ginecológica-obstétrica. G) Actividad física realizada durante la última semana y el último año, tanto en el trabajo como en faenas domésticas y ocio ${ }^{32,33}$. A cada actividad declarada se le asignó posteriormente el número de MET que se le atribuyen en el Compendio de Actividades Físicas de Ainsworth ${ }^{34}$. Los datos en relación al análisis del sedentarismo en la cohorte "CDC de Canarias" han sido publicados $\mathrm{ya}^{24}$. H) Dieta, explorada a través de un cuestionario de frecuencia y cantidad de alimentos (FFQ-CDC), validado para esta cohorte $^{35}$ y que es una actualización de la utilizada en la Encuesta Nutricional de Canarias (ENCA) en $1997^{36}$, el cual a su vez se originó de otro cuestionario anterior 37. I) Exposiciones ambientales y laborales. J) Hábitos higiénicos y relaciones sexuales.

Los casos prevalentes de cáncer y enfermedades cardiovasculares se recogieron 
por declaración del participante y se contrastaron entrecruzando las respuestas a una serie de preguntas dirigidas a confirmar su veracidad, como motivos de ingresos hospitalarios y consumo de fármacos. Está previsto, además, que en el seguimiento de la cohorte se contraste esta información con la historia clínica del participante, para lo cual se obtuvo consentimiento en el momento del reclutamiento. Para la diabetes mellitus se aceptó como diabético a toda persona que manifestara serlo y/o estuviera en tratamiento farmacológico-dietético prescrito por su médico, o a quien sin saberlo tuviera una glucemia en ayunas mayor de $125 \mathrm{mg} / \mathrm{dl}^{38}$; en este último caso, se realizó una segunda determinación basal de glucemia para confirmar el diagnóstico. Asimismo, se consideró que padecía hipertensión arterial (HTA) aquel participante que declarara serlo y/o estuviera en tratamiento para ello o quien presentara unas cifras de presión elevadas en el momento del estudio: a) Presión arterial sistólica $(\mathrm{PAS}) \geq 140 \mathrm{mmHg}$ o presión arterial diastólica (PAD) $\geq 90 \mathrm{mmHg}^{39}$.

Se consideró hipercolesterolémico al sujeto con diagnóstico previo si estaba en tratamiento hipolipemiante con dieta o fármacos, y también se aceptó como tal a aquel individuo que presentaba cifras séricas en ayunas ?240 mg/dl de colesterol total $^{40}$. Se entendió como bajos los valores de colesterol HDL ?40mg/dl en varones y ?50mg/dl en mujeres. Como definición del síndrome metabólico se utilizó la del ATPIII ${ }^{39}$ y la de la IDF ${ }^{41}$. El sedentarismo se midió según la clasificación de minutos de ocio activo (menos de 30 minutos) ${ }^{24}$. Se consideró fumador a quien declaró serlo. La exposición solar, el uso de protección frente a la misma y el consumo de gramos de alcohol se obtuvieron por declaración del participante.

Se midió la clase social de los sujetos mediante un índice de elaboración propia, basado en la propuesta realizada por el grupo de trabajo de la SEE y de la semFYC ${ }^{42}$, que incluye el grado de estudios alcanzado, la renta familiar per cápita, el índice de hacinamiento en el hogar, el sector de actividad laboral en el que trabaja el individuo y la situación laboral. El índice creado a partir de estas variables produjo una escala en un rango de valores entre 4 y 40 que se dividió en quintiles, lo cual permite comparación de exposiciones entre la clase social más rica y la más pobre.

La ancestría canaria se midió como la que presenta aquel participante que tanto él como sus padres y sus cuatro abuelos hubieran nacido en Canarias. Esta variable se considera de interés dado la supervivencia de la carga genética aborigen en una mayoría de la actual población canaria ${ }^{14}$.

Control de Calidad. Los datos recogidos en el cuestionario CDC han sido procesados de forma automática mediante lectura por escáner. Previamente, de forma aleatoria, se llevaron a cabo auditorías sobre el trabajo de producción de datos primarios realizado por encuestadores, enfermeras y técnicos de laboratorio, consistentes en la comprobación de un subconjunto de datos mediante repetición de su proceso de obtención. En caso de detectarse diferencias ostensibles se procedió a la revisión del lote completo de cuestionarios para ese día y persona, rectificándose el contenido de los cuestionarios antes de proceder a su vuelco en la base. Sobre la base de datos se realiza de forma periódica una revisión de consistencia a partir de los listados de las variables y la corrección de anomalías mediante consulta de los cuestionarios originales, que han sido almacenados en formato de imágenes TIFF. Un tercer mecanismo de control de la calidad se realiza a partir de la obtención de los histogramas para las variables numéricas continuas y diagramas de barras para las variables de escala ordinal, nominal y 
categórica, con el fin de detectar valores extremos aberrantes que son comprobados consultando los correspondientes cuestionarios originales.

Análisis de datos. Los datos obtenidos son analizados de forma transversal para conocer, en el momento del reclutamiento algunas características de interés de la población canaria, con especial énfasis en las prevalencias de exposición a FR para cáncer, diabetes mellitus y enfermedades cardiovasculares. Algunos de estos resultados preliminares se presentan en este artículo, resumiendo las variables cuantitativas mediante sus medias \pm desviación estándar y expresando las cualitativas según la frecuencia observada en sus categorías respectivos porcentajes. La comparación bivariada se efectuó con t-test para las variables cuantitativas y chi-cuadrado de Pearson para las cualitativas. Mediante modelos de regresión logística se ha estimado los riesgos relativos (odds ratios e intervalos de confianza $=\mathrm{OR}, \mathrm{IC}_{95 \%}$ ), ajustados por edad, de exposición a FR en el quintil mínimo frente al máximo de la variable clase social.

Seguimiento. El seguimiento se realiza con una frecuencia quinquenal, que incluye una actualización telefónica del cuestionario CDC para aquellos datos con posibilidad de modificación en ese lapso de tiempo y toma de muestras sanguíneas en los cortes correspondientes a los años $10^{\circ}$ y $20^{\circ}$ del seguimiento. El primero de estos contactos se está realizando durante el año 2008 y una vez finalizado se podrá medir por primera vez la incidencia. El seguimiento terminará por finalización del estudio, o pérdida del participante o, para cada una de las enfermedades específicas, con su padecimiento. Para fomentar la adherencia de los participantes y reducir las pérdidas durante el seguimiento, se les envía anualmente una tarjeta navideña que les recuerde que continúan enrolados en el estudio.

\section{RESULTADOS}

La cohorte reclutada (tabla 1) presenta una distribución por sexos próxima a la registrada en la población censada en Canarias con edad mayor de 18 años, de la cual es aproximadamente el 0'4\%. No obstante, en la isla de Gran Canaria existe sobrerepresentación del sexo femenino que responde a la estrategia de muestreo ya comentada. El diagrama de flujo del reclutamiento de los participantes (figura 1) muestra la organización del estudio en lo referente al muestreo, selección, trabajo de campo y procesamiento de datos.

En la tabla 2 se describe la distribución por sexo de las variables analizadas. Los

Tabla 1

Distribución de la cohorte CDC de Canarias y de la población de 18 a 75 años censada en el archipiélago, por sexo e isla, a 31 de diciembre de 2005

\begin{tabular}{|c|c|c|c|c|c|c|c|c|}
\hline & Tenerife & La Palma & La Gomera & El Hierro & Gran Canaria & Lanzarote & Fuerteventura & Canarias \\
\hline $\begin{array}{l}\text { Total } \\
\text { participantes }\end{array}$ & $2582(100 \%)$ & $395(100 \%)$ & $342(100 \%)$ & $464(100 \%)$ & $2245(100 \%)$ & $397(100 \%)$ & $293(100 \%)$ & $6.718(100 \%)$ \\
\hline $\begin{array}{l}\text { Total } \\
\text { población }\end{array}$ & $701.034(100 \%)$ & $78.800(100 \%)$ & $18.285(100 \%)$ & $8.682(100 \%)$ & $730.622(100 \%)$ & $96.781(100 \%)$ & $60.273(100 \%)$ & $1.694 .477(100 \%)$ \\
\hline $\begin{array}{l}\text { Mujeres } \\
\text { participantes }\end{array}$ & $1339(52 \%)$ & $227(57 \%)$ & $184(54 \%)$ & $239(51 \%)$ & $1423(63 \%)$ & $239(60 \%)$ & $159(54 \%)$ & $3.810(57 \%)$ \\
\hline $\begin{array}{l}\text { Mujeres } \\
\text { población }\end{array}$ & $355.559(51 \%)$ & $39.699(50 \%)$ & $9.006(49 \%)$ & $4.293(49 \%)$ & $367.444(50 \%)$ & $47.036(49 \%)$ & $28.379(47 \%)$ & $851.416(50 \%)$ \\
\hline $\begin{array}{l}\text { Hombres } \\
\text { participantes }\end{array}$ & $1234(48 \%)$ & $168(43 \%)$ & $158(46 \%)$ & $225(49 \%)$ & $822(37 \%)$ & $158(40 \%)$ & $134(46 \%)$ & $2.908(43 \%)$ \\
\hline $\begin{array}{l}\text { Hombres } \\
\text { población }\end{array}$ & $34.475(49 \%)$ & $39.101(50 \%)$ & $9.279(51 \%)$ & $4.389(51 \%)$ & $363.178(50 \%)$ & $49.745(51 \%)$ & $31.894(53 \%)$ & $843.061(50 \%)$ \\
\hline
\end{tabular}


Figura 1

Diagrama de flujo del reclutamiento de participantes de la cohorte "CDC de Canarias" (2000-2005)

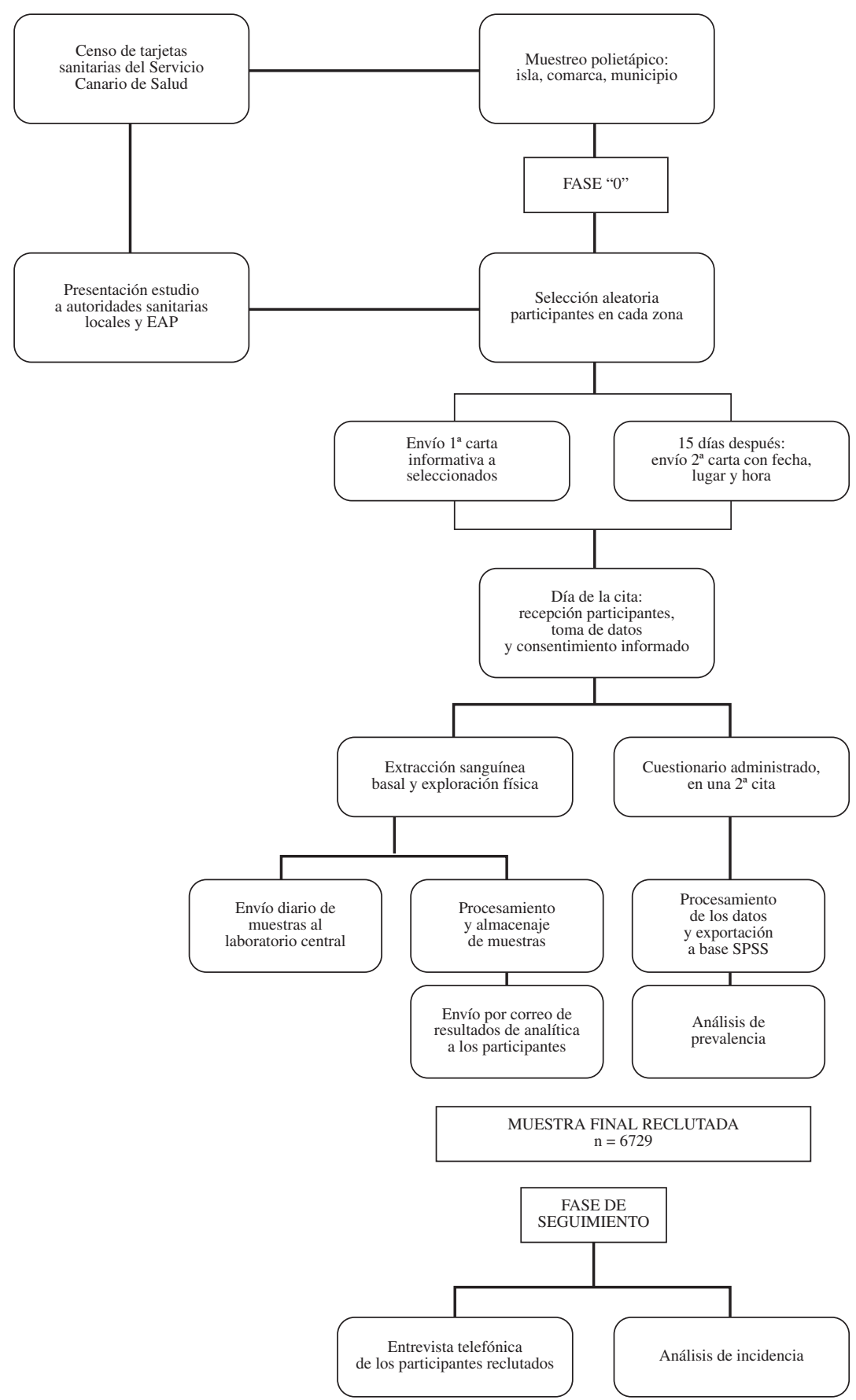


Tabla 2

Distribución por sexos de las variables antropométricas, biomédicas, bioquímicas y sociales de los participantes

\begin{tabular}{|c|c|c|c|}
\hline & Varones & Mujeres & $\mathbf{p}$ \\
\hline \multicolumn{4}{|l|}{ Tabla 2(a.) Antropometría } \\
\hline Edad (años) & $43 \pm 13$ & $43 \pm 13$ & 0,912 \\
\hline Peso $(\mathrm{Kg})$ & $81,6 \pm 13,8$ & $69,6 \pm 14,3$ & $<0,001$ \\
\hline Talla $(\mathrm{cm})$ & $172 \pm 7,3$ & $159,6 \pm 6,6$ & $<0,001$ \\
\hline IMC & $27,5 \pm 4,3$ & $27,5 \pm 5,6$ & 0,633 \\
\hline Cintura abdominal $(\mathrm{cm})$ & $95,5 \pm 11,5$ & $87,2 \pm 13,8$ & $<0,001$ \\
\hline Cintura pelviana $(\mathrm{cm})$ & $101,8 \pm 9,2$ & $101,8 \pm 12,5$ & 0,919 \\
\hline Ratio Abdomen/pelvis & $0,93 \pm 0,1$ & $0,85 \pm 0,1$ & $<0,001$ \\
\hline Ratio Abdomen/estatura & $0,55 \pm 0,0$ & $70,54 \pm 0,13$ & $<0,001$ \\
\hline \multicolumn{4}{|c|}{ Tabla 2(b). Variables biomédicas y bioquímicas } \\
\hline Frecuencia cardiaca (lpm) & $71 \pm 11$ & $75 \pm 10.2$ & $<0,001$ \\
\hline PAS (mmHg) & $127,3 \pm 17,7$ & $120,3 \pm 20$ & $<0,001$ \\
\hline PAD (mmHg) & $81 \pm 11$ & $75,7 \pm 13,4$ & $<0,001$ \\
\hline Glucosa (mg/dl) & $100,2 \pm 27,3$ & $93,9 \pm 23,9$ & $<0,001$ \\
\hline Colesterol total (mg/dl) & $204,2 \pm 42,5$ & $201,9 \pm 40,8$ & $<0,05$ \\
\hline Colesterol LDL (mg/dl) & $129,6 \pm 37,4$ & $125,6 \pm 35,9$ & $<0,001$ \\
\hline Colesterol HDL (mg/dl) & $46 \pm 12,3$ & $54,7 \pm 13,2$ & $<0,001$ \\
\hline Triglicéridos (mg/dl) & $140,8 \pm 101,5$ & $108,3 \pm 80$ & $<0,001$ \\
\hline Apolipoproteína-B (mg/dl) & $109,8 \pm 26$ & $104,3 \pm 28,7$ & $<0,05$ \\
\hline Leptina $(\mathrm{mg} / \mathrm{L})$ & $2,5 \pm 3,2$ & $11,3 \pm 8,8$ & $<0,001$ \\
\hline \multicolumn{4}{|l|}{ Tabla 2(c). Variables sociales. } \\
\hline Sedentarismo* & $1605(55)$ & $2720(71)$ & $<0,001$ \\
\hline Tabaquismo activo & $908(31)$ & $816(21)$ & $<0,001$ \\
\hline Ancestría canaria** & $2414(83)$ & $3119(82)$ & 0,118 \\
\hline Índice de clase social & $24,9 \pm 5,6$ & $23,3 \pm 7,4$ & $<0,001$ \\
\hline Ingesta calórica edad 30-45 (Kcal/día) & $2303,7 \pm 220,7$ & $1878 \pm 170,2$ & $<0,001$ \\
\hline Ingesta calórica edad 45-59 (Kcal/día) & $2086,7 \pm 194,6$ & $1765,9 \pm 165,8$ & $<0,001$ \\
\hline
\end{tabular}

Las variables cuantitativas se expresan en medias \pm desviación típica. Las cualitativas se expresan según las frecuencias absolutas observadas y frecuencias relativas $(\%) *$ Sedentarismo $=$ menos de 30 minutos diarios de actividad física moderada o intensa en tiempo de ocio. **Ancestría canaria = participante nacido en Canarias así como sus padres y sus cuatro abuelos.

varones presentaron valores significativamente más elevadas que las mujeres en la presión arterial, glucemia, lípidos séricos, tabaquismo, índice de clase social, ingesta calórica y los indicadores antropométricos de obesidad abdominal (cintura abdominal, RAP e IAE), pero no en el IMC y la cintura pelviana. Por el contrario, entre las mujeres se obtuvo valores significativamente más altos de frecuencia cardiaca, leptina, colesterol HDL y sedentarismo.

La tabla 3 muestra, estratificada por sexos y grupos de edad, la prevalencia de exposición a los FR cardiovascular estudiados así como de algunos factores asociados al desarrollo de cánceres (obesidad, exposición solar, consumo de alcohol y tabaquismo). Las diferencias entre sexos fueron significativas para la HTA ( $\mathrm{p}<0$ '001), ingesta excesiva de alcohol $(\mathrm{p}<0$ '001), diabetes $(\mathrm{p}=0$ '005), sobrepeso $(\mathrm{p}<0$ '001), colesterol HDL bajo ( $\mathrm{p}<0$ ’001), síndrome metabólico IDF $(\mathrm{p}<0$ '001) y uso de cremas de protección solar ( $\mathrm{p}<0$ ’001).

La tabla 4 muestra la prevalencia de cáncer declarada y la distribución por sexos y localización, de aquellos cánceres que superan el $1 \%$ en la muestra del CDC. Finalmente, en la tabla 5 se presentan las estimaciones del riesgo relativo, ajustado 
Tabla 3

Prevalencia de los factores de riesgo estudiados por grupos de edad

\begin{tabular}{|c|c|c|c|c|c|c|c|}
\hline \multicolumn{2}{|c|}{ Edad (años) } & $18-30$ & $>30-45$ & $>45-65$ & $>65$ & Total & $\mathbf{p}$ \\
\hline \multicolumn{8}{|l|}{ Varones } \\
\hline \multicolumn{2}{|l|}{ DM tipo 2} & $10(2)$ & $63(6)$ & $256(22)$ & $15(26.3)$ & $344(12)$ & $<0,001$ \\
\hline \multicolumn{2}{|l|}{ HTA $(>140 / 90)$} & $90(17)$ & $400(35)$ & $714(62)$ & $37(77)$ & $1241(43)$ & $<0,001$ \\
\hline \multicolumn{2}{|c|}{ Hipercolesterolemia* } & $45(8)$ & $331(29)$ & $527(46)$ & $29(52)$ & $932(32)$ & $<0,001$ \\
\hline \multicolumn{2}{|c|}{ Niveles bajos HDL-c*** } & $140(26)$ & $372(33)$ & $326(29)$ & $16(33)$ & $854(30)$ & $<0,05$ \\
\hline \multicolumn{2}{|c|}{ Sd. Metabólico-ATPIII } & $38(7)$ & $228(20)$ & 407 (36) & $24(50)$ & $697(24)$ & $<0,001$ \\
\hline \multicolumn{2}{|l|}{ Sd. Metabólico-IDF } & $64(12)$ & $357(31)$ & $611(54)$ & $33(69)$ & 1065 (37) & $<0,001$ \\
\hline \multicolumn{2}{|l|}{ Obesidad (IMC $\geq 30$ ) } & $67(12)$ & $257(22)$ & $438(38)$ & $13(28)$ & 775 (27) & $<0,001$ \\
\hline \multicolumn{2}{|c|}{ Sobrepeso $(25 \leq \mathrm{IMC}<30)$} & $178(35)$ & $508(48)$ & 485 (46) & $25(53)$ & $1196(45)$ & $<0,001$ \\
\hline \multicolumn{2}{|l|}{ Sedentarismo } & $223(41)$ & $627(55)$ & $719(62)$ & $36(63)$ & $1605(55)$ & $<0,001$ \\
\hline \multicolumn{2}{|l|}{ Tabaquismo activo } & $156(29)$ & $414(36)$ & $329(28)$ & $9(16)$ & $908(31)$ & $<0,001$ \\
\hline \multicolumn{2}{|l|}{ Clase social baja*** } & $30(6)$ & $98(10)$ & $228(22)$ & $30(57)$ & $386(15)$ & $<0,001$ \\
\hline \multirow{3}{*}{$\begin{array}{l}\text { Exposición solar } \\
\text { (hr/semana) }\end{array}$} & $0 \mathrm{~h}-5 \mathrm{~h}$ & $54(10)$ & $129(11)$ & $146(12)$ & $1(2)$ & $330(11)$ & \multirow[t]{3}{*}{0,130} \\
\hline & $5 \mathrm{~h}-15 \mathrm{~h}$ & $190(35)$ & $428(37)$ & $418(36)$ & $20(35)$ & $1056(36)$ & \\
\hline & $>15 \mathrm{~h}$ & $298(55)$ & $591(51)$ & $591(52)$ & $36(63)$ & $1527(52)$ & \\
\hline \multirow{2}{*}{$\begin{array}{l}\text { Uso de Protección } \\
\text { solar }\end{array}$} & Siempre & $243(45)$ & $534(47)$ & $294(27)$ & $3(5)$ & $1074(38)$ & \multirow[t]{2}{*}{$<0,001$} \\
\hline & Nunca & $175(32)$ & $418(37)$ & $674(61)$ & $50(89)$ & $1317(46)$ & \\
\hline \multirow{2}{*}{$\begin{array}{l}\text { Alcohol } \\
\text { gr/semana**** }\end{array}$} & Bajo & $238(44)$ & $565(50)$ & $590(51)$ & $21(37)$ & $1414(49)$ & \multirow[t]{2}{*}{$<0,001$} \\
\hline & Excesivo & $33(6)$ & $143(13)$ & 199 (17) & $6(11)$ & (13) & \\
\hline \multicolumn{8}{|l|}{ Mujeres } \\
\hline \multicolumn{2}{|l|}{ DM tipo 2} & $13(2)$ & $62(4)$ & $271(17)$ & $23(33)$ & $369(10)$ & $<0,001$ \\
\hline \multicolumn{2}{|l|}{ HTA $(>140 / 90)$} & $61(9)$ & 247 (17) & $876(57)$ & $48(75)$ & $1232(33)$ & $<0,001$ \\
\hline \multicolumn{2}{|c|}{ Hipercolesterolemia* } & $76(11)$ & $291(20)$ & $750(48)$ & $55(80)$ & $1172(31)$ & $<0,001$ \\
\hline \multicolumn{2}{|c|}{ Niveles bajos HDL-c*** } & $197(28)$ & $566(39)$ & $618(40)$ & $19(36)$ & $1400(37)$ & $<0,001$ \\
\hline \multicolumn{2}{|c|}{ Sd. Metabólico-ATPIII } & $36(5)$ & $208(14)$ & $637(41)$ & $27(51)$ & $908(24)$ & $<0,001$ \\
\hline \multicolumn{2}{|l|}{ Sd. Metabólico-IDF } & $47(7)$ & 279 (19) & $785(51)$ & $33(62)$ & $1144(31)$ & $<0,001$ \\
\hline Obesidad ( IMC $\geq 3$ & & $96(14)$ & $303(21)$ & $679(44)$ & $18(34)$ & $1096(29)$ & $<0,001$ \\
\hline Sobrepeso $(25 \leq \mathrm{IM}$ & & $139(21)$ & $440(31)$ & $574(38)$ & $24(45)$ & 1177 (33) & $<0,001$ \\
\hline Sedentarismo & & $429(61)$ & $1081(73)$ & $1158(74)$ & $52(75)$ & $2720(71)$ & $<0,001$ \\
\hline Tabaquismo activo & & $191(27)$ & $453(31)$ & $171(11)$ & $1(1)$ & $816(21)$ & $<0,001$ \\
\hline Clase social baja* & & $48(8)$ & $274(20)$ & $641(46)$ & $50(76)$ & $1013(29)$ & $<0,001$ \\
\hline Exposición solar & $0 \mathrm{~h}-5 \mathrm{~h}$ & $138(19)$ & $311(21)$ & $402(26)$ & $15(22)$ & $866(23)$ & $<0,001$ \\
\hline & $5 \mathrm{~h}-15 \mathrm{~h}$ & $392(55)$ & $808(55)$ & $855(55)$ & $46(67)$ & 2101(55) & \\
\hline & $>15 \mathrm{~h}$ & $178(25)$ & $356(24)$ & $307(20)$ & $8(12)$ & 849 (22) & \\
\hline Uso de Protección & Siempre & $543(78)$ & $1198(82)$ & $898(62)$ & $15(22)$ & $2654(72)$ & $<0,001$ \\
\hline & Nunca & $83(12)$ & $133(9)$ & 409 (28) & $40(59)$ & $655(18)$ & \\
\hline Alcohol & Bajo & $171(24)$ & $444(30)$ & $388(25)$ & $11(16)$ & 1014 (27) & $<0,05$ \\
\hline & Excesivo & $15(2)$ & $18(1)$ & $28(2)$ & $0(0)$ & $61(2)$ & \\
\hline
\end{tabular}

Los resultados se expresan según las frecuencias absolutas y frecuencias relativas $(\%)$. *Hipercolesterolemia $=$ Niveles de colesterol sérico $\geq 240 \mathrm{mg} / \mathrm{dl}$ o estar en tratamiento farmacológico o dietético. **Niveles bajos de HDL-c $=$ niveles séricos de colesterol HDL $\leq 40 \mathrm{mg} / \mathrm{dl}$ en varones y $\leq 50 \mathrm{mg} / \mathrm{dl}$ en mujeres. ATP $=$ Adult Treatment Panel III. IDF $=$ International Diabetes Federation. ****Quintil bajo del indicador de clase social. $* * * *$ Consumo de gramos de alcohol por semana: consumo bajo (menor de $175 \mathrm{gr} / \mathrm{semana}$ para varones y menor de $110 \mathrm{gr} / \mathrm{semana}$ para mujeres); consumo excesivo (mayor de 280 $\mathrm{gr} / \mathrm{semana}$ para varones y mayor de $168 \mathrm{gr} / \mathrm{semana}$ para mujeres). 
Tabla 4

Prevalencia (\%) declarada de cáncer: distribución por sexos y localización

\begin{tabular}{|c|c|c|}
\hline & Varones $(n=37)$ & Mujeres $(n=84)$ \\
\hline Prevalencia global & 1,3 & 2,2 \\
\hline Labio & 3 & 1,2 \\
\hline Lengua & 3 & 1,2 \\
\hline Colon & 5,4 & 1,2 \\
\hline Estómago & 3 & 2,4 \\
\hline Laringe & 13,5 & 1,2 \\
\hline Piel: Melanoma & - & 3,6 \\
\hline Piel: no melanoma. & 13,5 & 7,2 \\
\hline Linfomas y Tej. hematopoyético & 8,1 & 4,8 \\
\hline Mama & - & 26,2 \\
\hline Útero (endometrio y cérvix) & - & 20,3 \\
\hline Ovarios & - & 2,4 \\
\hline Vagina & - & 1,2 \\
\hline Próstata & 22 & - \\
\hline Tiroides & - & 1,2 \\
\hline Encéfalo & - & 2,4 \\
\hline Boca & 3 & - \\
\hline Óseo & 7,1 & - \\
\hline Cabeza, cara y cuello & - & 1,2 \\
\hline
\end{tabular}

Tabla 5

Estimación de los riesgos relativos, ajustados por edad (OR, IC95\%), de exposición a factores de riesgo cardiovascular y de cáncer en la clase social más baja frente a la más alta

\begin{tabular}{|c|c|c|c|c|}
\hline \multirow[t]{2}{*}{ Variable dependiente } & \multicolumn{2}{|c|}{ Hombres } & \multicolumn{2}{|c|}{ Mujeres } \\
\hline & OR $\left(\mathrm{IC}_{95 \%}\right)$ & $\mathbf{p}$ & OR $\left(\mathrm{IC}_{95 \%}\right)$ & $\mathbf{p}$ \\
\hline Sedentarismo & $1,30(0,98-1,73)$ & 0,07 & $1,60(1,26-2,04)$ & $<0,001$ \\
\hline Obesidad & $1,37(0,98-1,91)$ & 0,07 & $4,01(3,00-5,35)$ & $<0,001$ \\
\hline Obesidad Abdominal* & $1,75(1,29-2,37)$ & $<0,001$ & $4,70(3,64-6,06)$ & $<0,001$ \\
\hline Síndrome Metabólico ATP & $1,20(0,85-1,70)$ & 0,10 & $3,25(2,35-4,49)$ & $<0,001$ \\
\hline Síndrome Metabólico IDF & $1,34(0,99-, 82)$ & 0,06 & $2,42(1,84-3,20)$ & $<0,001$ \\
\hline Diabetes Mellitus & $2,06(1,29-3,28)$ & 0,002 & $2,81(1,74-4,53)$ & 0,001 \\
\hline Consumo excesivo de alcohol & $0,85(0,52-1,38)$ & 0,51 & $2,46(0,90-6,71)$ & 0,080 \\
\hline Exposición solar > 15 h./sem. & $2,35(1,48-3,73)$ & 0,000 & $1,63(1,16-1,29)$ & 0,005 \\
\hline No usa nunca crema protectora & $4,04(2,89-5,64)$ & $0-000$ & $4,00(2,74-5,83)$ & 0,000 \\
\hline Tabaquismo activo & $0,78(0,58-1,06)$ & $0-113$ & $1,47(1,11-1,94)$ & 0,008 \\
\hline
\end{tabular}

* Ratio Cintura abdominal/estatura $>0$ '55. ATP $=$ Adult Treatment Panel III. $\mathrm{IDF}=$ International Diabetes Federation

por edad, de exposición a FR cardiovascular y de cáncer en la clase social más baja frente a la más alta.

\section{DISCUSIÓN}

Nuestros resultados, extraídos de una amplia muestra de los adultos que residen en Canarias, describen una población con elevada prevalencia de FR, algunos descritos ya previamente por nuestro grupo 22,24 . Destaca la obesidad, cercana al $30 \%$ en ambos sexos, a la cual se añade un sobrepeso que en los varones alcanza al $45 \%$, lo cual no debe ser ajeno a las más altas prevalencias de diabetes y HTA en el sexo masculino. Por el contrario, en las mujeres es 
mayor el sedentarismo y en consonancia con esto presentan mayor prevalencia de niveles bajos de colesterol HDL. La exposición a los FR estudiados, entre ellos la pobreza, aumenta con la edad. La única excepción la constituye el tabaquismo, del cual ya es conocido que las mayores prevalencias se registran en edades jóvenes ${ }^{21}$.

Sumados la obesidad y el sobrepeso, los padecen aproximadamente el $70 \%$ de los adultos de Canarias, lo cual sitúa a estas islas a la cabeza de este problema en el país. Son resultados similares a los de EEUU, aunque allí es más elevada la prevalencia de obesidad mórbida ${ }^{43}$. En Europa se ha situado la prevalencia general de obesidad en un $10-15 \%{ }^{44}$, pero existen grandes variaciones entre países, por lo que estas tasas pueden llegar hasta el 30\% de obesos en la población adulta, con más de la mitad de la población en situación de sobrepeso. Los datos para España aportados por el grupo de expertos de la SEEDO en $2003^{45}$, mostraron una prevalencia general de obesidad del 14,5\%. Más recientemente la Encuesta Nacional de Salud (ENS) ${ }^{46}$, indica que la obesidad afectaría al 15,2\% de los adultos y que un $37,4 \%$ estaría en situación de sobrepeso $(44,4 \%$ de varones y $30,3 \%$ de mujeres). Con estas cifras de obesidad no es de extrañar que la prevalencia del síndrome metabólico que hemos encontrado sea también elevada, $24 \%$, dato muy similar al extraído de la ENCA ${ }^{23}$. Ello nos sitúa de nuevo en una situación semejante a la de EEUU, donde la prevalencia es del $24 \%{ }^{47}$.

La ingesta declarada muestra que los adultos canarios son una población sobrealimentada, particularmente los varones jóvenes. La ingesta energética diaria en el estudio "CDC de Canarias" supera lo registrado en ENCA una década antes ${ }^{48}$ y apunta una tendencia paralela al incremento experimentado por la obesidad en el archipiélago. Si tomáramos como referencia las ingestas recomendadas para una sociedad poco sedentaria estas cifras no serían exce- sivas, pero en la estimación de las necesidades energéticas de una población debe considerarse su gasto energético, que en Canarias es bajo por la elevada prevalencia de sedentarismo (55\% de los varones y $71 \%$ de las mujeres). La ENS $2006^{46}$ detectó un $40 \%$ de adultos sedentarios, incrementándose este porcentaje con la edad y entre las mujeres (37\% en los varones y $44 \%$ en las mujeres). Nuestros resultados indican que ésta es una Comunidad con baja actividad física.

La obesidad es el factor etiológico más directamente relacionado con la aparición de la diabetes tipo 2. Más del $85 \%$ de los diabéticos son del tipo 2 y, de ellos, el $90 \%$ presenta sobrepeso u obesidad ${ }^{49}$. En España, los estudios epidemiológicos sobre diabetes presentan diferencias metodológicas importantes, pero se ha estimado una prevalencia del 6,5\% en la población de 30-65 años y de un 10,3\% en grupos poblaciones de 30 a 89 años ${ }^{50}$. En la cohorte "CDC de Canarias" la prevalencia bruta de la diabetes tipo 2 fue del 11\%, (12\% en los varones y $10 \%$ en las mujeres). Tras estandarizar la edad, la diabetes resultó del 12,5\%. Otros trabajos que han estimado esta prevalencia en alguna localidad concreta del archipiélago, arrojan resultados muy parecidos a los nuestros, entre ellos un 13,2\% en el estudio de Telde ${ }^{51}$ y un $12,4 \%$ en el estudio de Guía ${ }^{52}$. Nuestro estudio es el primero en confirmar, en una amplia muestra con representación de todas las islas, que la población adulta de Canarias es la que sufre mayor prevalencia de diabetes tipo 2 en España. Esto era de esperar si se analiza la tasa de mortalidad por esta enfermedad, puesto que los varones canarios casi cuadruplican la mortalidad por diabetes de los residentes en Cantabria y triplican la de Madrid, mientras que las mujeres canarias triplican la mortalidad de Madrid y duplican las de muchas Comunidades ${ }^{19}$.

Los datos para la HTA y para la hipercolesterolemia tampoco son demasiado favo- 
rables. La prevalencia de la HTA detectada es del $38 \%$, lo que nos sitúa en niveles similares a la mayoría de los países desarrollados $(40 \%)$ y de la prevalencia española, que estaría en un $35 \%$ y llega hasta el $40 \%$ en edades medias de la vida ${ }^{53}$. Tal como era de suponer, la hipertensión es más frecuente entre los varones y aumenta de forma progresiva con la edad. En cuanto a la hipercolesterolemia los resultados vienen a situar a Canarias entre las Comunidades Autónomas con mayor prevalencia de este problema, lo cual ya se había apuntado en estudios no poblacionales ${ }^{54}$.

El cáncer constituye la principal causa de mortalidad en España, siendo más incidente en los hombres y destacando la frecuencia de cáncer colo-rectal, pulmón, mama, próstata y útero ${ }^{55}$. En nuestra cohorte, la prevalencia del cáncer se sitúa en un $2 \%$. Según la localización, entre los varones son más frecuentes los de próstata, piel (no melanoma) y laringe, seguidos por linfomas. Lógicamente, la ausencia de casos de cáncer de pulmón es atribuible a su alta letalidad, que le convierte en un cáncer de baja prevalencia pese a su elevada incidencia. Entre las mujeres la localización más frecuente sigue siendo la mama, seguida por útero (endometrio fundamentalmente) y piel (no melanoma). A pesar de que la autodeclaración es un procedimiento que resta sensibilidad a la medición de la prevalencia de cáncer, la distribución según localización y sexo es similar a lo detectado por el Registro de Cáncer de Canarias ${ }^{20}$ en esta población. Entre los FR para el cáncer, cabe destacar la elevada prevalencia de obesidad, la cual se asocia a algunos de los cánceres que hemos detectado como más prevalentes, tales como el de colon, mama o próstata ${ }^{56-59}$. Para los cánceres de piel el FR más importante es la exposición solar ${ }^{60}$, y las horas de exposición declaradas por nuestros participantes son elevadas, especialmente en los varones de 30 a 65 años, los cuales además son los que menos utilizan productos de protección solar. El consumo excesi- vo de alcohol se ha relacionado con cánceres del tracto gastrointestinal e hígado ${ }^{61}$. Los datos registrados en nuestro estudio muestran un $13 \%$ de bebedores excesivos entre los hombres, encontrándose la mayoría en edades activas de la vida, en torno a 45-65 años de edad. Datos extraídos de la $\mathrm{ENCA}^{48}$, mostraban también que ese grupo de edad es el que registra los mayores consumos medios.

Con respecto al tabaquismo, FR cardiovascular y de cáncer, la prevalencia encontrada (26\% global, $31 \%$ en varones y $21 \%$ de mujeres) es menor a la detectada en Canarias años atrás ${ }^{48,62}$, concordando ello con la tendencia a la disminución del consumo en el resto del país. La baja prevalencia de las mujeres se debe al escaso tabaquismo en las de más de 45 años de edad, pero en los grupos de 18 a 45 años la prevalencia de exposición es similar a la masculina.

En cuanto a la clase social, es conocido el gradiente inverso entre nivel socioeconómico y exposición a FR a lo largo de la vida 63 . Nuestros resultados señalan un predominio de la pobreza en las edades más avanzadas y en el sexo femenino, lo cual podría tener relación con el desfavorable perfil cardiovascular y de cáncer de las mujeres canarias. De hecho, esto se ve corroborado en general por mayores $\mathrm{OR}$ en el sexo femenino al confrontar clases pobres y ricas respecto a la exposición a factores causantes de estas enfermedades. Todo lo anterior, particularmente la alta frecuencia de exposición a obesidad, diabetes, síndrome metabólico y sedentarismo, explica que en la cohorte "CDC de Canarias" se detecte un riesgo cardiovascular que duplica al del estudio REGICOR ${ }^{21}$ y que las tasas de mortalidad por cardiopatía isquémica en la provincia de Las Palmas dupliquen a las de Gerona $^{16}$.

En definitiva, las Islas Canarias cuentan hoy con una población adulta sometida a frecuente exposición a FR para las enfermeda- 
des cardiovasculares, la diabetes y el cáncer, destacando especialmente la prevalencia de sobrepeso, obesidad, y sedentarismo. Si no se corrigen los hábitos de sobrealimentación y sedentarismo, es de esperar en las próximas décadas la aparición enfermedades cardiovasculares y diabetes en edades cada vez más precoces. En esta Comunidad se hace necesaria la puesta en marcha de políticas socio-sanitarias encaminadas a potenciar los hábitos de vida saludables, como el fomento de la actividad física en tiempo de ocio y la mejora de la alimentación.

\section{BIBLIOGRAFÍA}

1. Dawber TR, Meadors GF, Moore FE Jr. Epidemiological approaches to heart disease: the Framingham Study. Am J Public Health Nations. 1951; 41: 279-81.

2. Multiple risk factor intervention trial. Risk factor changes and mortality results. Multiple Risk Factor Intervention Trial Research Group. JAMA. 1982; 248: 1465-77.

3. Ascherio A, Rimm EB, Giovannuci EL, Spiegelman D, Stampfer M, Willet WC. Dietary fat and risk of coronary heart disease in men: cohort follow up study in the United States. BMJ. 1996; 313: 84-90.

4. Colditz GA, Manson JE, Hankinson SE. The Nurses' Health Study: 20-year contribution to the understanding of health among women. J Women Health. 1997; 6: 49-62.

5. Keys A, Mienotti A, Karvonen MJ, Aravanis C, Blackburn H, Buzina R, et al. The diet and 15-year death rate in the seven countries study. Am J Epidemiol. 1986; 124: 903-15.

6. Trichopoulou A, Costacou T, Bamia C, Trichopoulou D. Adherence to a Mediterranean Diet and Survival in a Greek Population. N Engl J Med. 2003; 348: 25599-08.

7. Riboli E, Kaaks R. The EPIC Project: rationale and study design. European Prospective Investigation into Cancer and Nutrition. Int J Epidemiol. 1997; 26 Suppl 1:S6-14.

8. Riboli E. The European Prospective Investigation into Cancer and Nutrition (EPIC): plans and progress. J Nutr. 2001;131: 170S-175S.
9. Grupo EPIC en España. Patrones de consumo y principales fuentes de ingestión de lípidos y ácidos grasos en la cohorte española del Estudio Prospectivo Europeo sobre Dieta y Cáncer (EPIC). Med Clin (Barc). 1999; 112: 125-32.

10. Perez G, Marrugat J, Sala J. Myocardial infarctation in Girona, Spain: attack, mortality rate and 28-day case fatality in 1998. Regicor Study Group. J Clin Epidemiol. 1993; 46: 1173-79.

11. Valdes S, Botas P, Delgado E, Alvarez F, Cadorniga FD. Population-based incidence of type 2 diabetes in northern Spain: the Asturias Study. Diabetes Care. 2007; 30: 2258-63.

12. Martínez-González MA, Sanchez-Villegas A, De IJ, Marti A, Martínez JA. Mediterranean diet and stroke: objectives and design of the SUN project. Seguimiento Universidad de Navarra. Nutr Neurosci. 2002; 5: 65-73.

13. Puig T, Varas C, Perez I, Lluis T, Balaguer I. Patrones de mortalidad en una cohorte de trabajadores seguida durante 28 años: estudio MANRESA. Rev Esp Cardiol. 2004; 57: 924-30.

14. Maca-Meyer N, Villar J, Perez-Mendez L, Cabrera A, Flores C. A tale of aborigins, conqueors and slaves: Alu insertion polymorphisms and the peopling of Canary Islands. Ann of Human Genetics. 2004; 68: 600-05.

15. Instituto Canario de Estadística. Disponible en: http://www.gobiernodecanarias.org/istac [última consulta, 30 de junio de 2008].

16. Plan de Salud de Canarias. Servicio Canario de Salud. Consejería de Sanidad y Consumo del Gobierno de Canarias. Disponible en http://www.gobiernodecanarias.org/sanidad/scs [última consulta, 30 de junio de 2008] .

17. Boix R, Cañellas S, Almazán J, Cerrato E, Meseguer M, Medrano MJ. Mortalidad cardiovascular en España. Año 2000. Bol Epidemiol Semanal (Ministerio de Sanidad y Consumo. España). 2003; 11: 241-244.

18. Centro Nacional de Epidemiología. Disponible en: http://www.isciii.es [última consulta, $30 \mathrm{de}$ junio de 2008].

19. Saavedra JM, Bello LM, Núñez D, Ortega P, y Medrano MJ. Mortalidad por enfermedad isquémica del corazón en las Islas Canarias. Errores en la certificación de la variable "residencia" de los fallecidos en las zonas turísticas. Bol Epidemiol Semanal (Ministerio de Sanidad y Consumo. España). 2001; 9: 161-163. 
20. Registro de Cáncer de Canarias. Disponible en: http://www.gobiernodecanarias.org/sanidad/scs [última consulta, 30 de junio de 2008].

21. Cabrera de León A, Almeida González D, Pérez Méndez LI, Carrillo Fernández L, Cueto Serrano M, Real Valcárcel E, et al. Tabaquismo en adolescentes. Prevalencia estimada mediante declaración y cotinina sérica. Gac Sanit. 1999; 13: 270-74.

22. Cabrera de León A, Rodríguez Pérez MC, del Castillo Rodríguez JC, Brito Díaz B, Pérez Méndez Ll, Muros de Fuentes M, et al. Estimación del riesgo coronario en la población de Canarias aplicando la ecuación de Framingham. Med Clín (Barc). 2006; 126: 521-26.

23. Álvarez León EE, Ribas Barba L, Serra Majem L1. Prevalencia del síndrome metabólico en la población de la Comunidad Canaria. Med Clin (Barc). 2003; 120:172-74.

24. Cabrera A, Rodríguez MC, Rodríguez LM, Anía B, Brito B, Muros M, et al. Sedentarismo: tiempo de ocio activo frente al porcentaje del gasto energético. Rev Esp Cardiol. 2007; 69: 244-50.

25. Dominguez S, Cabrera A, Bosa F, Perez L, Diaz L, Aguirre-Jaime A. High density lipoprotein colesterol increases with living altitude. Int $\mathrm{J}$ Epidemiology. 2000; 29: 65-70.

26. Consenso SEEDO'2000 para la evaluación del sobrepeso y la obesidad y el establecimiento de criterios de intervención terapéutica. Sociedad Española para el Estudio de la Obesidad (SEEDO). Med Clin (Barc). 2000; 115: 587-97.

27. Guía Práctica. Hipertensión Arterial en Atención Primaria. Sociedad Española de Medicina Familiar y Comunitaria. Segunda edición, 1999.

28. Toledo C, Pitters-Pérez S, Gonzalez-Acosta H, Carballo Trujillo I, Rodriguez Pérez MC, AguirreJaime A. CDC de Canarias: Estudio de Consanguinidad en la isla de El Hierro. Prog Diag Prenat. 2001; 13: 339-82.

29. Clasificación internacional de enfermedades, $9^{\text {a }}$ revisión. Modificación clínica. Actualización a la $4^{\mathrm{a}}$ edición. Ed Secretaría General Técnica del Ministerio de Sanidad y Consumo. Madrid 2001.

30. Clasificación Estadística Internacional de Enfermedades. CIE-10. Disponible en: http://www.medicin.com.ar [última consulta, 30 de junio de 2008].

31. Índice de clasificación Anatómica, Terapéutica y Química (ATC) con Dosis Diarias Definidas. Ed
WHO Collaborating Centre for Drug Statistics Methodology, Oslo 1999.

32. Elosua R, Marrugat J, Molina L, Pons S, Pujol E. On behalf of investigators of the MARATDON Group. Validation of the Minessota Leisure time physical activity questionnaire in Spanish men. J Epidemiol. 1994; 139: 1197-09.

33. Elosua R, Garcia M, Aguilar A, Molina L, Covas MI, Marrugat J. On behalf of investigators of the MARATDON Group. Validation of the Minnesota Leisure time physical activity questionnaire in Spanish women. Med Sci Sports Exerc. 2000; 32; 1431-37.

34. Ainsworth BE, Haskell WL, Whitt MC, Irwin ML, Swartz AM, Strath SJ, et al. Compendium of physical activities: an update of activity codes and MET intensities. Med Sci Sports Exerc. 2000; 32: S498-516.

35. Armando Aguirre-Jaime, Antonio Cabrera de León, Santiago Domínguez Coello, Carlos Borges Álamo, Lourdes Carrillo Fernández, Carlos Gavilán Batista, et al. Validación de un cuestionario de frecuencia de consumo de alimentos adaptado para el estudio y seguimiento de la población adulta de las Islas Canarias". Rev Esp Salud Pública, en prensa 2008.

36. Serra Majem L, Armas Navarro A, Ribas Barba L. Encuesta Nutricional de Canarias. Hábitos alimentarios y consumo de alimentos. Ed Servicio Canario de Salud, Santa Cruz de Tenerife. 1999; 1: $117-42$.

37. Martín-Moreno JM, Boyle P, Gorgojo L, Maisonneuve P, Fernández-Rodríguez JC, Salvini S, et al. Development and validation of food frequency questionnaire in Spain. Int J Epidemiol. 1993; 22: 512-19.

38. American Diabetes Association. Clinical Practice Recomendations 2002. Diabetes Care. 2002; 25 (Supl1).

39. National Cholesterol Education Program. Third Report of the National Cholesterol Education Program (NCEP) Expert Panel on Detection, Evaluation, and Treatment of High Blood Cholesterol in Adults (Adult Treatment Panel III) final report. Circulation. 2002; 106: 3143-421.

40. Medrano MJ, Pastor-Barriuso R, Boix R, del Barrio JL, Damián J, Alvarez R, et al.; investigadores del estudio ZACARIS. Coronary disease risk attributable to cardiovascular risk factors in the Spanish population. Rev Esp Cardiol. 2007; 60: $1250-56$. 
41. The IDF consensus worldwide definition of the metabolic syndrome. Disponible en: www.idf.org/webdata/docs/IDF_Metasyndrome_definition.pdf. 5 [última consulta, 30 de junio de 2008].

42. Grupo de trabajo de la Sociedad Española de Epidemiología y de la Sociedad Española de Medicina Familiar y Comunitaria. Una Propuesta de medida de la clase social. Aten Primaria. 2000; 25: $350-63$.

43. National Center for Health Statistics. Prevalence of overweight and obesity among adults: Unites States, 2003-2004. Disponible en: www.cdc.gov/nchs/products/pubs/pubd/hestats/overweight/ overwght_03.htm [última consulta, 30 de junio de 2008].

44. Varo JJ, Martínez-González MA, Martínez JA. Prevalencia de obesidad en Europa. An Sist Sanit Navar. 2002; 25: 103-08.

45. Aranceta J, Perez C, Serra L, Ribas L, Quiles J, Vioque J, et al. Prevalence of obesity in Spain: results of the SEEDO 2000 study. Med Clin (Barc). 2003; 120: 608-12.

46. Encuesta Nacional de Salud 2006. Resultados preliminares (marzo 2007). Ministerio de sanidad y Consumo. Gobierno de España. Disponible en: www.msc.es/estadEstudios/estadisticas/encuestaNacional/encuestaNac2006/ [última consulta, 30 de junio de 2008].

47. Ford ES, Giles WH, Dietz WH. Prevalence of the metabolic syndrome among US adults. JAMA. 2002; 287: 356-59.

48. Serra-Majem LI. Encuesta Nutricional de Canarias (1997-1998). Servicio Canario de Salud. Consejería de Sanidad y Asuntos Sociales. Gobierno de Canarias. Las Palmas de Gran Canaria, 2000. Disponible en: www.gobiernodecanarias.org/sani$\mathrm{dad} / \mathrm{scs} / 1 /$ plansalud/enca/tomo3/index.htm [última consulta, 30 de junio de 2008].

49. World Health Organization. Obesity: preventing and managing the global epidemic. WHO Obesity Technical Report Series 894. Geneva, Switzerland: World Health Organization; 2000. Disponible en: www.who.int. [última consulta, 30 de junio de 2008].

50. Goday A. Epidemiology of diabetes and its noncoronary complications. Rev Esp Cardiol. 2002; 55: $657-70$.

51. Boronat M, Varillas VF, Saavedra P, Suarez V, Bosch E, Carrillo A, et al. Diabetes Mellitus and impaired glucosa regulation in the Canary Islands (Spain): prevalence and associated factors in the adult population of Telde, Gran Canaria. Diabet Med. 2006; 23: 148-55.

52. de Pablos-Velasco PL, Martinez-Martin FJ, Rodriguez-Perez F, Ania BJ, Losada A, Betancor P. Prevalence and determinants of diabetes mellitus and glucosa intolerante in a Canarian Caucasican population-comparison of the $1997 \mathrm{ADA}$ and the 1985 WHO criteria. The Guia Study. Diabet Med. 2001; 18: 235-41.

53. Banegas JR. Epidemiología de la hipertensión arterial en España. Situación actual y perspectivas. Hipertensión. 2005; 22: 353-62.

54. Vegazo O, Civeira F, Banegas JR, Serrano PL, Jiménez FJ, Luengo E. Prevalencia de dislipemia en las consultas ambulatorias del Sistema Nacional de Salud: estudio HISPALIPID. Med Clín (Barc). 2006; 127: 331-34.

55. López-Abente Ortega G. La situación del cáncer en España. Ministerio de Sanidad y Consumo. Disponible en: www.msc.es/ciudadanos/enfLesiones/ enfNoTransmisibles/doc/situacionCancer.pdf [última consulta, 30 de junio de 2008].

56. Pischon T, Laman PH, Boeing H, Friedenreich C, Norat T, Tjonneland A et al. Body size and risk of colon and rectal cancer in the European Prospective Investigation into Cancer and Nutrition (EPIC). J Natl Cancer Inst. 2006; 98: 920-31.

57. Moore LL, Bradlee ML, Singer MR, Splansky GL, Proctor MH, Ellison RC et al. BMI and waist circumference as predictors of lifetime colon cancer risk in Framingham Study adults. Int J Obes Relat Metab Disord. 2004; 28: 559-67.

58. Calle EE, Rodriguez C, Walker-Thurmond K, Thun MJ. Overweight, obesity, and mortality from cancer in a prospectively studied cohort of U.S. adults. N Engl J Med. 2003; 348:1625-38.

59. Cohen SS, Palmieri RT, Nyante SJ, Koralek DO, Kim S, Bradshaw P et al. Obesity and screening for breast, cervical, and colorectal cancer in women: a review. Cancer. 2008; 112:1892-04.

60. Rigel DS. Cutaneous ultraviolet exposure and its relationship to the development of skin cancer. J Am Acad Dermatol. 2008; 58: S129-32.

61. Pelucchi C, Gallus S, Garavello W, Bosetti C, La Vecchia C. Alcohol and tobacco use, and cancer risk for upper aerodigestive tract and liver.Eur $\mathrm{J}$ Cancer Prev. 2008; 17: 340-44. 
62. Encuesta de Salud de Canarias 2004. Servicio Canario de la Salud. Gobierno de Canarias. Disponible en: www.gobiernodecanarias.org/sanidad/scs1/ [última consulta, 30 de junio de 2008].
63. Mackenbach JP, Stirbu I, Roskam AJ, Schaap MM, Menvielle G, Leinsalu M, et al.; European Union Working Group on Socioeconomic Inequalities in Health. Socioeconomic inequalities in health in 22 European countries. N Engl J Med. 2008; 358: 2468-81. 